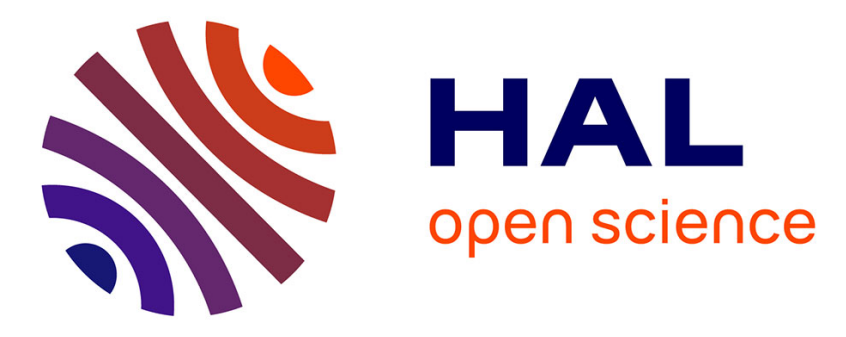

\title{
L'Index critique littéraire du romantisme et du naturalisme
}

Jean-Baptiste Amadieu

\section{To cite this version:}

Jean-Baptiste Amadieu. L'Index critique littéraire du romantisme et du naturalisme. Cahiers de l'Association internationale des études françaises (CAIEF), 2010, 62, p. 325-341. halshs-01315494

\section{HAL Id: halshs-01315494 \\ https://shs.hal.science/halshs-01315494}

Submitted on 4 Jun 2016

HAL is a multi-disciplinary open access archive for the deposit and dissemination of scientific research documents, whether they are published or not. The documents may come from teaching and research institutions in France or abroad, or from public or private research centers.
L'archive ouverte pluridisciplinaire $\mathbf{H A L}$, est destinée au dépôt et à la diffusion de documents scientifiques de niveau recherche, publiés ou non, émanant des établissements d'enseignement et de recherche français ou étrangers, des laboratoires publics ou privés. 


\title{
№62 CAHIERS DE L'ASSOCIATION INTERNATIONALE DES ÉTUDES FRANÇAISES
}

\author{
RENAN DANS SON TEMPS ET LE NÔTRE
}

L'ÉCRIVAIN CRITIQUE D'ART

CENSURE ET CRÉATION LITTÉRAIRE

\section{Mai $2010 \quad N^{\circ} 62$}




\title{
L'INDEX CRITIQUE LITTÉRAIRE DU ROMANTISME ET DU NATURALISME
}

\author{
Communication de M. Jean-Baptiste AMADIEU \\ (École normale supérieure, Paris)
}

au LXI Congrès de l'Association, le 8 juillet 2009

Les archives de la Congrégation de l'Index récemment ouvertes nous révèlent les motifs pour lesquels Balzac, Sand, Lamartine, Hugo, Sue, Dumas, Stendhal, Flaubert ou encore Zola furent inscrits dans l'Index librorum prohibitorum. Les examens ou "vota» rédigés par les consulteurs de la Congrégation constituent un aussi curieux que précieux laboratoire de critique littéraire institutionnelle. Les censeurs ecclésiastiques ont-ils perçu à l'occasion des procès d'œuvres déterminées une doctrine homogène caractéristique de la génération, en particulier le romantisme et le naturalisme? Les deux grandes esthétiques du siècle, si elles n'échappent pas aux rapporteurs de la Congrégation chargée par le Saint-Siège de la censure des livres, leur posent deux difficultés. Le premier paradoxe concerne l'objet de la censure : quelques propositions extraites d'un ouvrage ou bien une doctrine ? L'autre difficulté concerne la nature de la doctrine. Si un tribunal ecclésiastique paraît préparé à une évaluation théologique ou morale, est-il compétent pour se prononcer en matière de doctrines littéraires? Les censeurs romains mentionnent pourtant le romantisme et le naturalisme dans leurs vota comme deux doctrines incompatibles avec la foi de l'Église. 
Toutes sommaires que soient les considérations des rapporteurs de l'Index sur le romantisme, elles signalent l'écart entre l'anthropologie et la théologie romantiques et l'enseignement ecclésial. La doctrine romantique est régulièrement assimilée à une hérésie de l'époque, connue sous le nom d'indifférentisme et appliquée par Grégoire XVI à Lamennais. À la fin du siècle, à l'occasion des quatre censures de Zola, les rapporteurs rapprochent le naturalisme esthétique d'une doctrine condamnée, le naturalisme philosophique, c'est-àdire la négation du surnaturel. On juge alors le naturalisme esthétique solidaire du matérialisme athée, et comme son application dans la littérature, en s'exposant au risque de confusion lexicale favorisée par l'homonymie.

\section{LE ROMANTISME DOCTRINAL}

Paul Bénichou définit l'âge romantique comme "une époque de doctrine [...]. Ce travail d'idées s'étend aux œuvres littéraires elles-mêmes ; jamais auparavant la poésie et les ouvrages de fiction n'ont prétendu être plus gravement pensants (1) ». La floraison de religiosités s'accompagne d'une volonté de régénérer le christianisme, de l'épurer de ses dogmes, de ses rites, de ses impératifs disciplinaires et de ses institutions, sinon de tentatives pour formuler de nouvelles conceptions spirituelles. Les travaux de Paul Bénichou ont constaté l'émergence d'une nouvelle figure de poète, d'un véritable magistère voire d'un sacerdoce poétique, mais insubordonné à tout dogme. La condamnation de Lamennais avertit que l'Église n'est pas disposée à accepter les régénérations qu'on lui propose de l'intérieur, ni a fortiori à se réjouir des nouvelles spiritualités extravagantes qui foisonnent de toute part. Plusieurs œuvres déférées devant le tribunal de

(1) Paul Bénichou, Le Temps des prophètes, Paris, Gallimard, 1977, p. 565. 
l'Index sont interdites au motif que les conceptions religieuses développées sont irrecevables en bonne orthodoxie. C'est notamment le cas du Voyage en Orient et de Jocelyn de Lamartine, de Lélia et de Spiridion de Sand, et du Livre mystique de Balzac. Chaque examen définit certaines caractéristiques de l'hétérodoxie romantique: le mysticisme avec Le Livre mystique de Balzac ; l'indifférentisme à partir du votum sur le Voyage en Orient de Lamartine; le prophétisme avec quelques examens de Sand.

\section{Le Livre Mystique de Balzac, ou le mysticisme hétérodoxe}

Le votum de Michele Zecchinelli s'ouvre par une copieuse introduction qui présente la trilogie du Livre mystique de Balzac composée des Proscrits (1831), de Louis Lambert (1832) et de Séraphîta (1835), puis il examine successivement chacun des trois titres. Son rapport permet de se faire une idée des censures doctrinales des religiosités nouvelles développées dans les œuvres littéraires. La première partie du votum expose la théorie du Livre mystique (2) :

Bien qu'il semble que l'auteur écrive de simples romans, il apparaît néanmoins clairement, qu'avec une méthode artificielle, il se prend à développer un système religieux dont luimême ne voudrait pas paraître partisan, mais qu'il propose cependant comme préférable aux autres, y compris au catholicisme. Dans la préface, il atteste qu'on y personnifie les sentiments et les systèmes humains. Et donc Séraphîta est l'expression du «mysticisme», c'est-à-dire du «Christianisme dans son principe pur (3)» (Préface, p. 6).

Et là, subitement, il fait connaître quelle sorte de religion, ou de pensée religieuse comme il l'appelle, il va nous indiquer dans son mysticisme, en disant (p. 6 de la préface) que

(2) Le texte qui suit est une traduction de ACDF (abr. de Archivio della Congregazione per la Dottrina della fede, Palazzo del Santo Officio, Città del Vaticano), Index, Protocolli 1838-1841, f. 561r-562r.

(3) Balzac, Le Livre mystique, Paris, Werdet, t. I (Les Proscrits et Histoire intellectuelle de Louis Lambert), p. VII. 
« [d]octrine des Premiers Chrétiens, religion des Anachorètes du Désert, le Mysticisme ne comporte ni gouvernement, ni sacerdoce (4) ».

Ce ne sera cependant pas une surprise, si faisant apparaître sa Séraphîta comme une créature prédestinée et élevée à la connaissance de l'infini, elle est transformée après sa mort en un séraphin. Pourtant, il la représente comme non baptisée ; elle affirme même qu'elle n'a pas besoin de baptême, étant suffisamment pure, parce qu'élue avant sa naissance. Il la représente en outre morte sans aucun secours religieux et sans l'assistance d'un vrai ministre consacré, et cependant élevée immédiatement au plus haut des cieux, au seul moyen de la prière.

Il est aisé de noter que l'auteur entend faire du mysticisme presque un concentré et une quintessence de tous les mystères du paganisme, qui admet audacieusement des renouvellements et presque des remaniements de saint Jean dans son apocalypse.

«Ce qui jusqu'à présent manquait au mysticisme était la forme, la poésie. Quand saint Pierre a montré les clefs du Paradis et l'enfant Jésus dans les bras d'une vierge, la foule a compris! Et la religion catholique a existé. Le rusé saint Pierre, homme de haute politique et de gouvernement, a eu raison sur saint Paul, ce lion des Mystiques, comme saint Jean en est l'aigle (5). »

Zecchinelli censure d'abord l'idée d'une pureté mystique dévoyée par l’Église. Selon le romancier, la doctrine ecclésiastique se serait éloignée de l'esprit évangélique et chrétien. Le sacerdoce et les sacrements seraient des institutions tardives. Le censeur s'en prend ensuite à l'apport de traditions religieuses non chrétiennes au mysticisme: l'idée de profit qu'auraient tiré Moïse et Jean des mysticismes indien, grec et égyptien affaiblit l'idée de Révélation. Balzac hisse aussi Swedenborg au rang d' "évangéliste » alors que ses Principia rerum naturalium (1734) furent mis à

(4) Ibid.

(5) Balzac, Le Livre mystique, op. cit., t. I, p. XII-XIII. 
l'Index en 1738. Zecchinelli revient enfin à l'idée d'une pureté mystique dévoyée par l'Église: Balzac oppose Pierre, dont se réclame le Saint-Siège, à Paul et Jean qui seraient les autorités de la religion mystique. Pour reprendre la typologie de Péguy, Pierre a dévoyé la mystique chrétienne en politique.

Le mysticisme exposé par Balzac suppose une antinomie inacceptable pour un censeur romain entre une doctrine chrétienne primitive et l'institution ecclésiastique qui s'en est éloignée. Cette religiosité sape ainsi la légitimité des rites, des sacrements et des dogmes institués par l'Église. L'hérésie romantique consiste en une critique du monopole ecclésiastique en matière de communication avec le divin. Peut-on se sauver hors de l'Église? Le romantisme religieux envisage des alternatives à l'exclusivité salvifique de l'Église, notamment la nature et le cœur.

\section{Lamartine et la censure de l'indifférentisme}

Dans son examen du Voyage en Orient, le cardinal Polidori dénonce de nombreux aspects hétérodoxes de l'œuvre. Il juge que Lamartine s'abandonne aux élans de son cœur, de ses intuitions, plutôt qu'à l'étude raisonnée. Il rattache cette méthode à l'« esprit romantique » de l'auteur. Polidori associe le romantisme à la fantaisie, à l'ignorance de l'enseignement scripturaire ou magistériel. La « hardiesse » romantique construit des croyances d'intuition, poétise un contenu religieux au sens étymologique.

La critique majeure que Polidori adresse au Voyage en Orient est le reproche d'indifférentisme. Le terme apparaît à l'occasion de la censure d'un extrait où Lamartine, alors qu'il se trouve en Terre Sainte, médite sur le christianisme :

Absorbé dans sa méditation sur ce lieu saint, l'auteur continue à exposer les impressions qu'il en éprouve, quelle que soit l'opinion religieuse qu'on ait, et toujours animé par l'indifférentisme, il distingue le christianisme qui est attaché à la lettre, aux dogmes de sa mère, du christianisme philosophique, 
qui est dit de l'esprit, préférant le second parce que l'esprit vivifie, tandis que la lettre tue. Les sentiments, que l'auteur expose, conviennent au déiste, au naturaliste, à l'hétérodoxe quelconque; ce sont des lignes empreintes de rationalisme religieux. On y confond celui qui croit en la divinité du Christ, et celui qui ne le connaît que comme homme, et tous s'imaginent chrétiens : mais celui qui ne professe pas la divinité du Christ n'est certainement pas chrétien (6).

La distinction de la lettre qui tue et de l'esprit qui vivifie, reprise de saint Paul (2Co, III, 6), pourrait donner des gages scripturaires à la réflexion censurée si le rapporteur n'y voyait de l'exégèse de complaisance. En amont de ce jugement, Polidori attribue la distinction des deux christianismes à l'esprit de l'auteur "animato sempre per l'indifferentismo ». Le mysticisme de Balzac s'appuyait sur une distinction semblable entre l'esprit évangélique et la lettre chrétienne.

L'indifférentisme que Polidori discerne chez Lamartine substitue à la foi théologale et raisonnée le sentiment religieux. Lamartine se dit chrétien non pas par nécessité de se sauver, ni par grâce divine, mais parce que le christianisme est conforme à sa sensibilité, atténuant la nature impérative et exclusive de la foi chrétienne :

Les religions sont une affaire d'instinct, non de raisonnement, déclare l'auteur, et, sous prétexte de professer le christianisme, il croit qu'il faut l'alléguer, parce que la fibre de mon cour est chrétienne, parce que les sympathies de mon âme et de mon esprit sont pour cette doctrine, parce que je vis de l'air de mon temps sans prévoir de quoi vivra l'avenir. Injurieuses raisons pour une religion toute divine, seule vraie, et qui trouve seulement dans la parole divine le motif pour qu'on la professe. Avec une telle maxime, quiconque professe une religion différente serait justifié. Là où seul compte l'instinct, où l'on n'agit pas, sinon pour seconder son propre talent, où la raison de se décider sur une chose

(6) Trad. de ACDF, Index, Protocolli 1836-1838, f. 264v. 
de tant d'importance est la fibre du cœur, où l'on n'attend rien d'autre que la sympathie d'esprit, il manque l'unité de principe, il manque la liberté, il manque la foi donnée par la grâce, et il est indifférent d'avoir une religion plutôt qu'une autre (7).

La profession chrétienne de Lamartine repose sur une adhésion de cœur et d'instinct, au lieu d'un acte de foi. Le cœur peut cependant s'épancher vers n'importe quelle croyance. La justification affective de l'appartenance au christianisme est assimilée à une forme d'indifférentisme. D'une part Lamartine critique le christianisme dogmatique, " de la lettre », dans lequel le censeur semble se reconnaître ; d'autre part il prône un christianisme philosophique et affectif. Ces deux traits définissent l'écart du Voyage en Orient avec la doxa catholique, laquelle récuse les rapports au divin soit immédiats, soit médiatisés hors de l'Église. L'hétérodoxie de Lamartine tient au refus de l'infaillibilité du magistère ecclésiastique et de l'exclusivité salvifique de l'Église. L'homme peut accéder à la divinité par le moyen de la nature ou par sa conscience et son cœur.

\section{La mission prophétique de Sand}

Deux censures de Sand éclairent d'autres aspects du romantisme vu par l'Index : l'idéalisme philosophique, le sacre de l'auteur et l'influence de Lamennais. Le votum de Vincenzo Tizzani sur Lélia (novembre 1840) tente une définition de la philosophie romantique et indique le lien de Sand avec Lamennais; l'examen que rédige Augustin Theiner sur Jacques et Leone Leoni (mars 1841) traite de la dangereuse mission dont Sand se croit investie.

Le votum de Tizzani sur Lélia de Sand est le premier texte de l'Index à proposer une définition du romantisme comme doctrine philosophique. Le censeur note une certaine filiation avec la philosophie des Lumières : le roman- 
tisme est le dernier déguisement que revêt la soi-disant philosophie. Le Saint-Siège définit régulièrement les nouvelles hérésies par d'anciennes. Les deux systèmes partagent un même refus de la philosophie pérenne réaliste. Le romantisme est en effet un idéalisme : au sens philosophique, il modèle des êtres idéaux et propose une spiritualité transcendantale ; au sens commun, cet idéalisme est une doctrine chimérique qui abandonne et détruit toute idée de réalité. L'idéalisme romantique conduit :

- à une croyance religieuse affective, une "religione del cuore », laquelle remplace la religion chrétienne. Polidori l'entendait de la sorte dans sa censure du Voyage en Orient ;

- à une pratique morale monstrueuse : en dépit de la séduction qu'exerce cette philosophie, elle aboutit à une fin différente de son dessein originel. L'application de ses principes dans la vie morale crée un homme monstrueux. Le malentendu tient à l'idéalisme illusoire et éloigné d'une juste compréhension de l'homme et du monde.

Augustin Theiner, autre censeur de Sand, consacre trois pages de son votum sur Jacques et Leone Leoni à une sorte de biographie intellectuelle et morale de l'écrivain (8). Il commence par commenter de façon énigmatique le pseudonyme de Sand: «titulo che caratterizza assai lo spirito infausto di questa miserabile autrice (9) ». Theiner fait allusion à l'assassinat du comte August de Kotzebue, écrivain et premier président du Conseil du Roi, à Berlin le 23 mars 1819, par un jeune étudiant allemand du nom de Karl-Ludwig Sand. Cette coïncidence est pour lui l'occasion d'une comparaison frappante entre les homonymes. Karl-Ludwig fut initié à l'illuminisme allemand et, entre la baronne au nom menteur et l'assassin illuminé et ennemi des trônes, le censeur voit une parentèle spirituelle :

(8) ACDF, Index, Protocolli 1838-1841, f. 537r sqq.

(9) Ibid., f. 537r. Trad. : « titre qui caractérise très bien l'esprit funeste de ce misérable auteur ». 
Voici en peu de mots la parentèle spirituelle de cette dame avec M. Sand déjà mentionné. Elle-même a assumé, de son côté, la triste charge d'une mission semblable à celle de ce malheureux jeune homme susnommé, mission rendue plus terrible et plus infernale encore, pour être non seulement destinée à renverser les trônes, mais aussi à détruire la religion et l'Église, et à bannir tous les principes sains, tant sociaux que moraux. Il n'y a pas d'écrivain contemporain qui ait su manier une telle mission avec autant de prestige d'esprit que Mme Dudevant. Elle a inventé un art complètement nouveau en France, à savoir l'art de représenter les objets les plus horribles, les plus obscènes, les plus impies avec le langage le plus séduisant, le plus libre et le plus sublime, si l'on peut qualifier ainsi un langage qui s'avilit dans la peinture des plus atroces obscénités du monde social. [...] On ne doit donc pas s'étonner si, aux pieds de cette dame, nous voyons même, dans notre siècle abasourdi, les sieurs de Chateaubriand, de Lamartine et de Lamennais, assis pour jouir de ses sentences spirituelles, et la vénérer comme la grande pythonisse de notre temps (10).

Sand incarne le prophétisme romantique. Sa mission ne vient pas d'un mandat de l'Église, elle se l'est attribuée ellemême. La comparaison de la mission de George Sand avec celle de l'assassin de Kotzebue suit une gradation : elle est d'abord semblable, avant de devenir pire. Karl-Ludwig Sand s'était donné pour simple mission d'attenter au pouvoir civil en assassinant l'un de ses représentants; son homonyme se donne un dessein plus dangereux, subvertir les principes de la foi, de la morale et de la société. À la fin du paragraphe, Theiner voit dans George Sand la «gran Pitonessa del nostro tempo », une prophétesse, une voyante, en référence à l'oracle d'Apollon pythien. Elle est prophétesse en ce qu'elle annonce un avenir où les principes religieux, moraux et sociaux du passé seront renversés ; et elle est prophétesse aussi au sens où elle exerce cette mission avec une autorité spirituelle puissante et inquiétante. 


\section{D'UN NATURALISME À L'AUTRE}

Un malentendu sur naturalisme peut prêter à confusion, puisqu'outre sa signification esthétique d'école cherchant un style naturel et approprié aux réalités sociales qu'on aborde à la manière des sciences expérimentales, le terme a aussi une acception ecclésiastique. Dans cette dernière, le terme est associé au fatalisme, à la négation de la spiritualité de l'âme et au socialisme. Il bannit la religion de la société civile et rejette la doctrine et l'autorité de la Révélation divine. Dans le Syllabus, il admet pour seule réalité la nature et son mot d'ordre serait: " Il faut nier toute action de Dieu en l'homme et dans le monde (11) ». Le dictionnaire de Pierre Larousse en donne la définition courante : « les naturalistes sont des athées, mais des athées définis, qui reconnaissent à la matière une ou plusieurs forces essentielles, soumises à des lois immuables, cause et règle de l'univers. »

Rien ne semble a priori rapprocher les deux acceptions : le naturalisme littéraire est une tendance stylistique (un langage naturel qui ne rejette pas la crudité quand elle est adéquate au sujet) et thématique (le social) ; le naturalisme philosophique un système qui place la nature comme premier principe à la place de Dieu. Dans l'esprit des deux censeurs de Zola, son naturalisme est un matérialisme et un athéisme. Est-ce une simple confusion lexicale? Ou n'y a-t-il pas des convergences entre les deux acceptions, du moins chez Zola ?

La première censure de son œuvre est due à Luigi Tripepi qui examine Lourdes en 1894. Deux traits caractérisent les romans de Zola, écrit-il, l'immoralité et l'impiété. Ce sont de dignes effets du naturalisme et du matérialisme professés par l'auteur, et de cet obscène vérisme, qui en est la conséquence en soi, et en même temps la méthode littéraire d'un homme qui a écrit L'Assommoir, La Faute de l'abbé Mouret, Nana, La Terre, La Fortune des Rougon (12).

(11) Denzinger, $n^{\circ} 2902$.

(12) Trad. de ACDF, Index, Protocolli 1894-1896, n 87, p. 1. 
À lire Tripepi, l'immoralité et l'impiété des romans de Zola auraient trois sources. L'une est clairement philosophique : le matérialisme, et c'est, avec l'athéisme, l'un des synonymes les plus courants du naturalisme philosophique. Une autre est clairement littéraire : le vérisme ou réalisme, qui est conçu comme une méthode littéraire ; c'est, pour simplifier, le naturalisme esthétique. La troisième source est précisément le naturalisme, dont on ignore si Tripepi l'entend au sens philosophique ou littéraire. Seule certitude, le vérisme, ou naturalisme littéraire, est une conséquence du matérialisme, le naturalisme philosophique. Le censeur articule les deux acceptions par une relation de cause à conséquence; cependant, si le matérialisme conduit au réalisme esthétique, il n'y a pas de certitude que le réalisme suppose nécessairement le matérialisme. L'examen de Tripepi semble d'abord poser que Zola est matérialiste, avant d'en déduire qu'il est donc vériste.

Lourdes a déclenché l'intérêt de la Congrégation pour l'œuvre de Zola. Quelques semaines après la mise à l'Index du roman, on demande à Alessio Boccasso d'examiner les opera omnia du romancier. Devant l'ampleur de la tâche, il s'épargne la lecture intégrale des Rougon en choisissant d'analyser la théorie générale de Zola d'après ses déclarations d'intention, de constater ensuite qu'elle est bien appliquée dans quelques-unes de ses fictions (dont $\mathrm{La}$ Terre, qui est peu propice à favoriser l'aménité d'un censeur d'Eglise), pour conclure que l'œuvre entière découle de cette théorie. Avec une telle méthode, il suffit de montrer l'hétérodoxie des principes théoriques pour justifier la proscription de l'œuvre dans son ensemble. La première partie du votum est donc consacrée à l'examen de la théorie intellectuelle et esthétique de Zola. Boccasso donne à cette partie le titre d'essais critiques. En six pages, il cite et commente des extraits du Roman expérimental, du Naturalisme au théâtre et du court essai intitulé Du roman. Il en ressort une théorie esthétique naturaliste qui, tout en se 
recommandant de la science, s'appuie sur des postulats philosophiques incompatibles avec l'orthodoxie romaine.

La première partie du votum de Boccasso est répartie en neuf rubriques, qui présentent la théorie de Zola comme une succession de principes qui s'enchaînent les uns aux autres. Les commentaires y sont rares, Boccasso se borne à présenter une synthèse des enjeux théoriques sans ressentir la nécessité d'en montrer les atteintes au dogme ou à la morale, tant elles semblent évidentes. En voici le résumé (13) :

I-II. (p. 1-2) Le postulat fondamental et " critère suprême » de l'écrivain selon Zola est le déterminisme, selon lequel l'homme est lié à l'hérédité et au milieu social. La physiologie découvrira un jour les mécanismes individuels. La tâche de l'écrivain est d'étudier les interactions entre l'individu et la société.

III. (p. 2-3) Zola distingue le déterminisme du fatalisme, ce qui laisse le censeur perplexe : affirmer une telle distinction ne la prouve pas. La distinction établie par Zola provient de Claude Bernard. Mais le censeur ne s'étend pas sur la distinction et revient à la définition du déterminisme qu'il compare au concept de raison suffisante chez Leibniz, et qu'il trouve négateur de la liberté humaine :

Si le déterminisme domine tout, et s'explique et se propage par disposition héréditaire et sous l'influence de circonstances données, l'homme n'est plus libre: il est vertueux ou vicieux selon les circonstances (14).

Le déterminisme s'attaque ainsi à la morale : en altérant voire en abolissant la liberté, Zola détruit toute possibilité

(13) Le chiffre romain donne le numéro de la rubrique dans le votum, et il est suivi des numéros de pages. Références du document: ACDF, Index, Protocolli 1894-1896, n 129.

(14) Trad. de ibid. 
de poser un acte moral ou immoral; dès lors l'idée de péché perd son sens.

IV. (p. 3-4) Les sciences progressent en révélant les lois fixes du vivant. Boccasso cite le raisonnement de Zola qui s'achève par cette affirmation scandaleuse pour un homme de foi :

puisque des savants [...] démontrent maintenant que des lois fixes régissent le corps humain, on peut annoncer, sans crainte de se tromper, l'heure où les lois de la pensée et des passions seront formulées à leur tour (15).

Pour le censeur, cette proposition aggrave les précédentes, où il était simplement question d'une détermination des comportements, en voyant dans le déterminisme le «dominatore eziandio degli atti intellettuali e morali (16)».

V. (p. 4-5) Les écrivains naturalistes s'attachent à l'observation des « lois » de la nature, à la différence des idéalistes qui « admettent des influences mystérieuses échappant à l'analyse ».

VI. (p. 5) Le progrès des sciences de l'observation, notamment en cosmographie et géologie, a porté « un si terrible coup aux fables des religions ».

VII. (p. 5) Le romancier naturaliste est semblable au savant décrivant le fruit de son observation. Pour un censeur catholique, cette affirmation est suspecte dans la mesure où la littérature prétendant à la description et non à l'édification, évolue indépendamment de toute valeur

(15) Zola, Le Roman expérimental (Le Roman expérimental, Lettre à la jeunesse, Le Naturalisme au théâtre, L'Argent dans la littérature, Du roman, De la critique, La République et la littérature), Paris, G. Charpentier et Cie, 1890, p. 15.

(16) ACDF, Index, Protocolli 1894-1896, n 129, p. 4. 
morale. L'objectivité est synonyme de neutralité, et d'une amoralité qui ouvre la voie à l'immoralité. Pour Zola, au contraire, il serait immoral pour l'écrivain de trahir les faits et une nature humaine qui n'est pas absolument honnête, mais comporte « un fonds de bête humaine chez tous ».

VIII. (p. 5-6) Face à ce constat anthropologique, l'écrivain pourrait se satisfaire d'un silence pudique sur les aspects corrompus de la nature humaine :

La voix moyenne serait de se taire, ou de raconter les faits éminemment moraux, en négligeant les autres. Mais ce pisaller ne plait pas à Zola, et il le dit contraire au caractère et à la mission de l'écrivain (17).

Zola compare l'écrivain à un magistrat dont le devoir est de tout savoir pour pouvoir tout juger.

IX. (p. 6) Les précédentes rubriques se voulaient une simple relation de la pensée de Zola, sans y ajouter de commentaire ou de jugement, à l'exception de la troisième. L'évaluation de l'œuvre selon le dogme et la morale semble suffisamment évidente pour que Boccasso ne s'y attarde pas : «Il detto è più che sufficiente per giudicare della immoralità dello Zola scrittore (18). » Une ultime citation de l'œuvre est censée montrer «tutta la perfidia di lui come scrittore» :

On est très coupable, quand on écrit mal ; en littérature, il n'y a que ce crime qui tombe sous mes sens, je ne vois pas où l'on peut mettre la morale, lorsqu'on prétend la mettre ailleurs. Une phrase bien faite est une bonne action (19).

C'est là le principal sujet de grief, la proposition qui résume les incriminations contre les écrits de Zola, «la

(17) Trad. de ibid., p. 5-6.

(18) Ibid., p. 6. Trad. : "Ce qui vient d'être dit est plus que suffisant pour juger de l'immoralité de Zola écrivain. »

(19) Zola, Le Roman expérimental, op. cit., p. 364. 
chiave per condannare ciò che ha scritto ": l'autonomie de l'esthétique vis-à-vis de l'éthique.

L'exposé que présente Boccasso des principes directeurs de l'œuvre de Zola permet de préciser la philosophie sousjacente à l'esthétique. Cette philosophie est-elle naturaliste? Boccasso n'use pas du terme, ni pour parler de la pensée postulée par Zola, ni de son esthétique. La censure de Boccasso reproche au déterminisme affiché de Zola, où l'on peut voir du naturalisme philosophique, d'exclure la liberté de son anthropologie en supposant que la nature suive des lois, y compris dans les comportements humains qu'on peut expliquer par l'atavisme et le milieu social ; et au naturalisme esthétique d'exclure la moralité de son esthétique en chargeant l'écrivain de transcrire les « lois de la nature ».

Censure-t-on le naturalisme? Les vota de Tripepi et Boccasso diffèrent dans leur méthode. Tripepi condamne le naturalisme esthétique comme une conséquence et une traduction littéraire du matérialisme et de l'athéisme, les deux synonymes du naturalisme philosophique; dans sa première censure (l'examen de Lourdes), il affirme ce lien entre les deux acceptions du naturalisme plus qu'il ne le démontre. Il conçoit d'abord les deux naturalismes comme un tout homogène, sans considérer que cette solidarité ne tient qu'à un mot. Puis vient le votum des opera omnia par Boccasso, qui montre l'articulation entre la philosophie erronée de Zola et son esthétique, usant de l'idée de nature humaine déterminée comme passerelle entre les deux domaines. Néanmoins Boccasso a la prudence de ne jamais utiliser le terme de naturalisme, ni dans un sens ni dans l'autre. Ce qu'il présente comme le critère esthétique de Zola se conforme au naturalisme esthétique ; et on peut considérer la pensée déterministe influencée par Claude Bernard comme une forme ou une application du naturalisme philosophique. D'autre part la censure de Boccasso montre qu'il n'est pas nécessaire de recourir à l'homogénéité de la philosophie et de l'esthétique de Zola pour le juger hétérodoxe : le seul critère littéraire de 
la transcription exhaustive du réel autonome de toute considération morale suffit à le soupçonner. En 1896, puis en 1898, Tripepi examine ensuite les deux autres volumes de la trilogie des Trois Villes au fur et à mesure de leur parution: Rome puis Paris. Progressivement, il abandonne l'acception de naturalisme au sens de vérisme, pour ne retenir que l'athéisme. Dans sa censure de Paris, il utilise le terme en un sens philosophique pour parler de Pierre Froment se déliant de ce qui pouvait encore l'attacher à l'Évangile :

auprès de Marie Couturier, il est heureux de s'adonner aux théories du pur naturalisme, d'oublier les craintes de la vie future, la pensée de la mort, de s'abandonner à la félicité sur la terre, sans se soucier du catholicisme, religion de la mort (20).

Le naturalisme, le «pur » naturalisme, est la doctrine qui libère l'homme des craintes sur l'au-delà, et lui propose le bonheur ici-bas, par opposition au catholicisme. Le terme désigne l'incroyance. En somme, le censeur qui se donnait le projet de censurer le naturalisme esthétique y renonce, et celui qui réalise ce projet n'ose pas user du concept ; celui qui aurait voulu condamner le naturalisme esthétique au nom de sa solidarité avec le naturalisme philosophique ne démontre pas l'articulation des deux, et celui qui paraît la démontrer, ne juge pas que cette solidarité soit nécessaire pour réprouver le naturalisme esthétique.

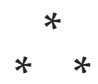

L'Index censure les mauvaises croyances romantiques avant de condamner l'incroyance naturaliste. L'Index saisit les égarements doctrinaux propres au romantisme

(20) Trad. de ACDF, Index, Protocolli 1897-1899, doc. 178, p. 6. 
comme un individualisme religieux, entendu au sens où la foi est un sentiment individuel immanent et où l'individu crée sa propre religion ou participe à son élaboration, d'où il résulte une variété d'énoncés extravagants, émancipés des dogmes et de la médiation de l’Église pour accéder à la connaissance du surnaturel. Le Saint-Siège juge inacceptable qu'on considère les sacrements comme non nécessaires au salut, la doctrine ecclésiastique comme tardive et éloignée de l'esprit évangélique, le cœur comme origine et justification de la foi, les poètes comme des prophètes ayant pour mission de réformer la foi, la morale et la société.

La censure du naturalisme présente-t-elle des analogies avec celle du romantisme? Les censeurs du romantisme voient dans cette tendance littéraire un terreau favorable aux pensées religieuses dissidentes du catholicisme voire du christianisme; la veine littéraire romantique favorise cette hétérodoxie par le culte du sentiment, le mélange du sacré et du profane, le goût de l'originalité et de la hardiesse. L'Index associe donc le romantisme à une doctrine religieuse, une mauvaise foi. Il n'en va pas de même pour le naturalisme. En dépit d'une réduction un peu hâtive chez Tripepi du naturalisme littéraire au naturalisme philosophique, on distingue la censure de l'incroyance de la censure de la méthode littéraire ; si la première condamne les énoncés au nom de la doctrine, la seconde dénonce plutôt l'immoralité de la volonté de dire toute la nature.

Jean-Baptiste AMADIEU 